\title{
Impulse Noise Removal in Digital Images
}

\author{
J.Harikiran \\ Assistant Professor, \\ Department of IT, GIT, \\ GITAM University
}

\author{
B.Saichandana \\ Assistant Professor, \\ Department of CSE,GIT, \\ GITAM University
}

\author{
B.Divakar \\ Student, \\ Department of I.T,GIT, \\ GITAM University
}

\begin{abstract}
This paper introduces the concept of image fusion of filtered noisy images for impulse noise reduction. Image fusion is the process of combining two or more images into a single image while retaining the important features of each image. Multiple image fusion is an important technique used in military, remote sensing and medical applications. Five different filtering algorithms are used individually for filtering the image captured from the sensor. The filtered images are fused to obtain a high quality image compared to individually denoised images. In-order to better appraise the noise cancellation behavior of our fusion technique from the point of view of human perception, an edge detection is performed using canny filter for the fused image. Experimental results show that this method is capable of producing better results compared to individually denoised images.
\end{abstract}

\section{KEYWORDS}

Impulse Noise, Image Enhancement, Image Restoration, Image Processing, Image Fusion.

\section{INTRODUCTION}

Order statistics filters exhibit better performance as compared to linear filters when restoring images corrupted by impulse noise. Impulse noises are short duration noises which degrade an image. They may occur during image acquisition, due to switching, sensor temperature. They may also occur due to interference in the channel and due to atmospheric disturbances during image transmission.

The goal of the filtering action is to cancel noise while preserving the integrity of edge and detail information. In this paper, we propose a novel technique for impulse noise reduction. In our technique, first an image in captured by a sensor. The image is filtered in parallel with five different smoothing filters. The denoised images obtained from five different filters are fused them to obtain a high quality image free from impulse noise.

The rest of the paper is organized as follows. Section II analyzes the impulse noise in images, , Section III presents the image fusion technique with five different smoothing filters, Section IV presents the experimental results and finally Section $\mathrm{V}$ report conclusions.

\section{IMPULSE NOISE IN IMAGES}

Impulse noise [4] corruption is very common in digital images. Impulse noise is always independent and uncorrelated to the image pixels and is randomly distributed over the image. Hence unlike Gaussian noise, for an impulse noise corrupted image all the image pixels are not noisy, a number of image pixels will be noisy and the rest of pixels will be noise free. There are different types of impulse noise namely salt and pepper type of noise and random valued impulse noise.

In salt and pepper type of noise the noisy pixels takes either salt value (gray level -225) or pepper value (grey level -0) and it appears as black and white spots on the images. If $p$ is the total noise density then salt noise and pepper noise will have a noise density of $p / 2$.This can be mathematically represented by (1)

$y_{i j}=\left\{\begin{array}{c}\text { zero or } 255 \text { with probability } p \\ x_{i j} \text { with probability } 1-p\end{array}\right.$

where $y_{i j}$ represents the noisy image pixel, $p$ is the total noise density of impulse noise and $x_{i j}$ is the uncorrupted image pixel. At times the salt noise and pepper noise may have different noise densities $p_{1}$ and $p_{2}$ and the total noise density will be $p=p_{1}+p_{2}$.

In case of random valued impulse noise, noise can take any gray level value from zero to 225 . In this case also noise is randomly distributed over the entire image and probability of occurrence of any gray level value as noise will be same. We can mathematically represent random valued impulse noise as in (2).

$y_{i j}=\left\{\begin{array}{l}n_{i j} \text { with probability } p \\ x_{i j} \text { with probability } 1-p\end{array}\right.$

where $n_{i j}$ is the gray level value of the noisy pixel.

\section{IMAGE FUSION TECHNIQUE}

The process of combining two or more images into a single image while retaining the important features of each image is called image fusion. In this paper, the filtered images from five different smoothing algorithms are fused to obtain a high quality denoised image. The block diagram of the process is shown in figure 1.

The five different smoothing algorithms used in our technique are described as follows: 


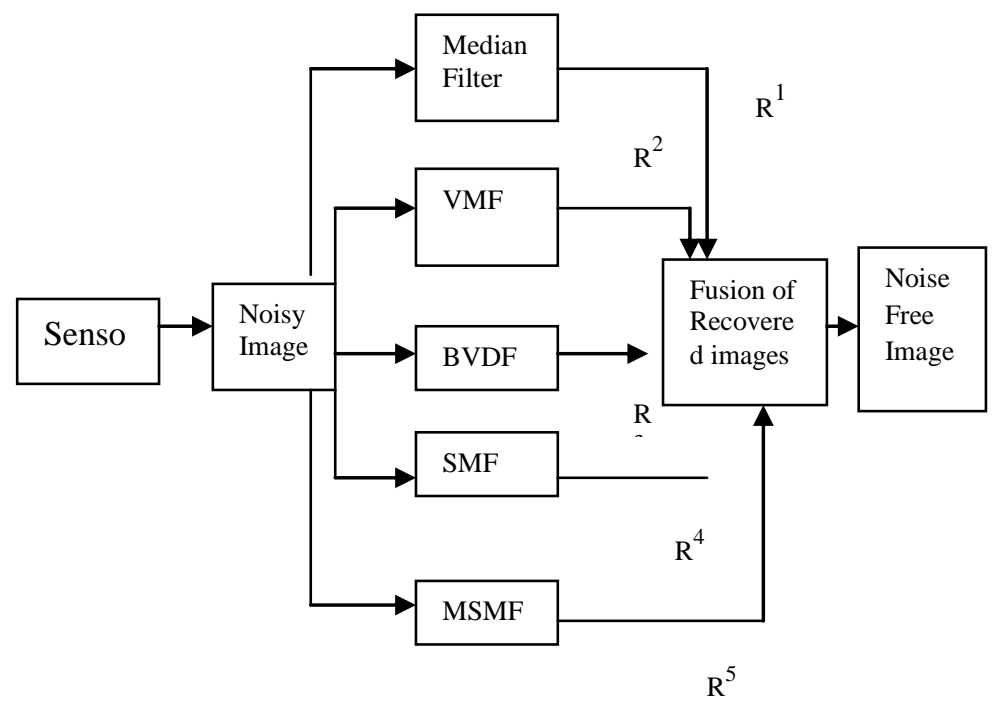

Figure 1: Block diagram of image fusion technique

Order-static filters are nonlinear filters whose response is based on the ordering (ranking) the pixels contained in the image area encompassed by the filter, and then replacing the value of the center pixel with the value determined by the ranking result. The best known filter in this category is the median filter, which as the name implies, replaces the value of the pixel by the median of the intensity values in the neighborhood of that pixel defined in (3). The pixel with the median magnitude is used to replace the pixel in the signal studied.

\section{$\operatorname{MEDIANFILTER}\left(\mathrm{x}_{1}, \mathrm{x}_{2}, \ldots \ldots \mathrm{x}_{\mathrm{N}}\right)=$ $\operatorname{MEDIAN}\left(\mathrm{x}_{1}, \mathrm{x}_{2}, \ldots \ldots \mathrm{x}_{\mathrm{N}}\right)$}

The median filter is more robust with respect to the presence of noise.

In the Vector median filter (VMF) [1] for the ordering of the vectors in a particular kernel or mask a suitable distance measure is chosen. The vector pixels in the window are ordered on the basis of the sum of the distances between each vector pixel and the other vector pixels in the window.

The sum of the distances is arranged in the ascending order and then the same ordering is associated with the vector pixels. The vector pixel with the smallest sum of distances is the vector median pixel. The vector median filter is represented as

$\mathrm{X}_{\mathrm{VMF}}=$ vectormedian (window)

If $\delta_{\mathrm{i}}$ is the sum of the distances of the $\mathrm{i}^{\text {th }}$ vector pixel with all the other vectors in the kernel, then

$\delta_{\mathrm{i}}=\sum_{j=1}^{N} \Delta\left(\mathrm{X}_{\mathrm{i}}, \mathrm{X}_{\mathrm{j}}\right)$

where $(1 \leq \mathrm{i} \leq \mathrm{N})$ and $\mathrm{X}_{\mathrm{i}}$ and $\mathrm{X}_{\mathrm{j}}$ are the vectors, $\mathrm{N}=9$.
$\Delta\left(\mathrm{X}_{\mathrm{i}}, \mathrm{X}_{\mathrm{j}}\right)$ is the distance measure given by the $L_{1}$ norm or the city block distance which is more suited to non correlated noise. The ordering may be illustrated as

$\delta_{1} \leq \delta_{2} \leq \delta_{3} \leq \ldots, \leq \delta_{9}$

and this implies the same ordering to the corresponding vector pixels i.e.

$X_{(1)} \leq X_{(2)} \leq \ldots, \leq X_{(9)}$

where the subscripts are the ranks. Since the vector pixel with the smallest sum of distances is the vector median pixel, it will correspond to rank 1 of the ordered pixels, i.e.,

$X_{V M F}=X_{(1)}$

For Basic Vector Directional Filter (BVDF) [5], Let W be the processing window of size $n$, and let $x_{i}, i=1,2, \ldots n$ be the pixels in $\mathrm{W}$. Let also the vector valued image function at pixel $x_{i}$ is denoted as $f_{i}$. Let $\alpha_{i}$ correspond to $f_{i}$.

$\alpha_{\mathrm{i}}=\sum_{j=1}^{n} \mathrm{~A}\left(\mathrm{f}_{\mathrm{i}}, \mathrm{f}_{\mathrm{j}}\right)$

where $A\left(f_{i}, f_{j}\right)$ denotes the angle between $f_{i}$ and $f_{j}$. An ordering of the $\alpha_{i}$ s $s$

$\alpha_{(1)} \leq \alpha_{(2)} \leq \ldots \ldots \ldots \alpha_{(m)} \leq \ldots \ldots \alpha_{(n)}$

implies the same ordering to the corresponding $\mathrm{f}_{\mathrm{i}}$ 's

$\mathrm{f}^{(1)} \leq \mathrm{f}^{(2)} \leq \ldots \ldots \ldots \mathrm{f}^{(\mathrm{m})} \leq \ldots \ldots \mathrm{f}^{(\mathrm{n})}$

The first term in (10) constitutes the output of the BVDF

$\operatorname{BVDF}\left[\mathrm{f}_{1}, \mathrm{f}_{2}, \ldots \ldots \mathrm{f}_{\mathrm{n}}\right]=\mathrm{f}^{(1)}$

The Spatial median filter (SMF) [2] is a uniform smoothing algorithm with the purpose of removing noise and fine points of image data while maintaining edges around larger shapes. The SMF is based on the spatial median quantile function which is a $\mathrm{L}_{1}$ norm metric that measures the difference between two vectors. The spatial depth between a point and a set of points is defined by

$\mathrm{S}_{\mathrm{depth}}\left(\mathrm{X}, \mathrm{x}_{1}, \mathrm{x}_{2}, \ldots \ldots \mathrm{x}_{\mathrm{N}}\right)=1-\frac{1}{N-1}\left\|\sum_{i=1}^{N} \frac{X-x i}{\|\mathrm{X}-\mathrm{X} i\|}\right\|$

Let $\mathrm{r}_{1}, \mathrm{r}_{2}, \ldots . \mathrm{r}_{\mathrm{N}}$ represent $\mathrm{x}_{1}, \mathrm{x}_{2}, \ldots . . \mathrm{x}_{\mathrm{N}}$ in rank order such that

$$
\begin{aligned}
& S_{\text {depth }}\left(\mathrm{r}_{1}, \mathrm{x}_{1}, \mathrm{x}_{2}, \ldots \ldots \mathrm{x}_{\mathrm{N}}\right) \\
\geq & \mathrm{S}_{\text {depth }}\left(\mathrm{r}_{2}, \mathrm{x}_{1}, \mathrm{x}_{2}, \ldots . . \mathrm{x}_{\mathrm{N}}\right) \\
\geq & S_{\text {depth }}\left(\mathrm{r}_{\mathrm{N}}, \mathrm{x}_{1}, \mathrm{x}_{2}, \ldots . . \mathrm{x}_{\mathrm{N}}\right)
\end{aligned}
$$

and let $r_{c}$ represent the center pixel under the mask. Then $\operatorname{SMF}\left(\mathrm{x}_{1}, \mathrm{x}_{2}, \ldots \ldots \mathrm{x}_{\mathrm{N}}\right)=\mathrm{r}_{1}$ 
In the Modified Spatial Median Filter (MSMF) [2], first calculate the spatial depth of every point within the mask and then sort these spatial depths in descending order. After the spatial depth of each point within the mask is computed, an attempt is made to use this information to first decide if the mask's center point is an uncorrupted point. If the determination is made that a point is not corrupted, then the point will not be changed. If the point is corrupted, then the point is replaced with the point with the largest spatial depth.

We can prevent some of the smoothing by looking for the position of the center point in the spatial order statistic. Let us consider a parameter $\mathrm{P}$ (where $1 \leq \mathrm{P} \leq \mathrm{N}$, where $\mathrm{N}$ represents numbers of points in the mask), which represents the estimated number of original points under a mask of points. If the position of the center mask point appears within the first $P$ ranks of the spatial order statistic, then we can argue that while the center point is not the best representative point of the mask, it is likely to be original data and should not be replaced. The MSMF is defined by

$\operatorname{MSMF}\left(T, x_{1}, x_{2}, \ldots . . x_{N}\right)= \begin{cases}r_{c} & c \leq P \\ r_{1} & c>P \quad(15)\end{cases}$

We generated five denoised images using the above smoothing algorithms. By fusing the best features of these five denoised images, a high quality image is obtained. The fusion of the filtered images is given by

$$
\mathrm{F}=\sum_{\mathrm{i}} \zeta \mathrm{R}^{\mathrm{i}} \text { where } \mathrm{i}=1,2,3,4,5
$$

$\mathrm{R}^{1}$ is the median filtered image, $\mathrm{R}^{2}$ vector median filtered image, $\mathrm{R}^{3}$ vector directional filtered image, $\mathrm{R}^{4}$ spatial median filtered image and $\mathrm{R}^{5}$ modified spatial median filtered image. $\zeta$ is the fidelity factor. The fusion criterion depends on the fidelity factor of the image. For a heavily noised image the fidelity factor $\zeta$ will be smaller compared to a lightly noised image and hence we fuse the images, each image contributes to the recovered image depending on its noise density. A lightly noised image contributes more compared to a heavily noised image. This helps to obtain a high quality fused image. We have taken fidelity factor $\zeta=0.2$.

\section{EXPERIMENTAL RESULTS}

This section presents the simulation results illustrating the performance of the proposed fusion technique. The test image employed here is the true color image "parrot" with $290 \times 290$ pixels. The salt and pepper noise is added into the image with noise density 0.4. The noise model was computer simulated. All filters considered operate using $3 \times 3$ processing window. The performance of filters was evaluated by computing the mean square error (MSE) between the original image and filtered image as follow:

$$
\operatorname{MSE}=\left(\frac{1}{M}\right) \sum_{n \in F}\left(\mathrm{I}(\mathrm{x}, \mathrm{y})-\mathrm{I}^{1}(\mathrm{x}, \mathrm{y})\right)^{2}
$$

Where $\mathrm{F}$ denotes the set of $\mathrm{M}$ processed pixels, $\mathrm{I}(\mathrm{x}, \mathrm{y})$ denotes the vector pixel value in the original image and $I^{1}(x, y)$ denotes the vector pixel value in the filtered image.
Figure 4 shows the results of filtering the color image parrot which is corrupted by impulse noise.

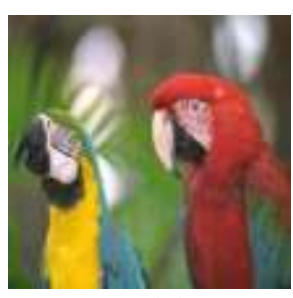

(a)

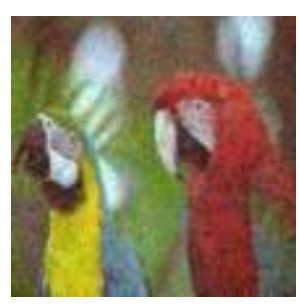

(c)

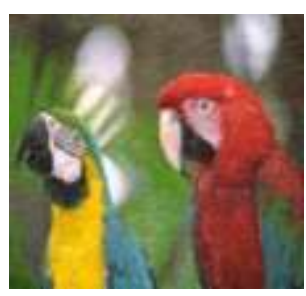

(e)

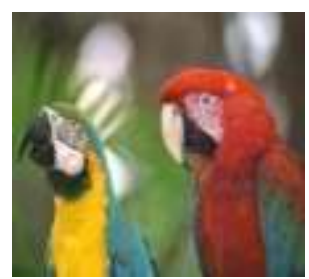

(g)

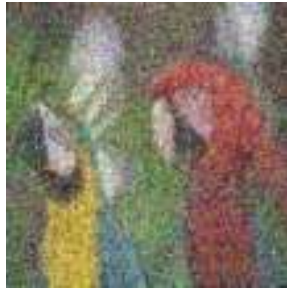

(b)

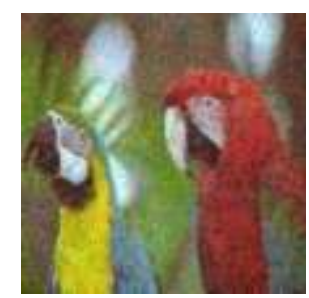

(d)

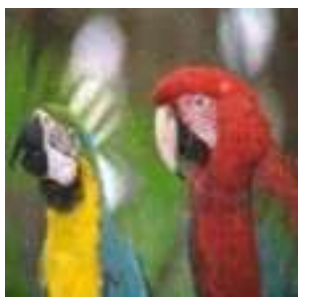

(f)

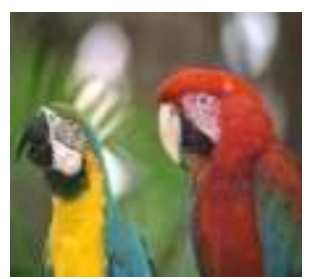

(h)
Figure 4 a) original image, b) impulse noise image corrupted by noise density $0.4, \mathrm{c}$ ) median filter, d) VMF, e) BVDF, f) SMF g) MSMF with $\mathrm{P}=4$ and h) Fused image.

Table (1) shows the results of MSE values of our fusion method, compared with five different smoothing filters. As it can be seen, the minimum mean square error (MSE) was obtained by our proposed approach.

Table 1: MSE values

\begin{tabular}{|l|l|}
\hline IMAGES & MSE \\
\hline Noise image & 40.3457 \\
\hline Median filter & 24.3675 \\
\hline Vector Median Filter & 20.2896 \\
\hline BVDF & 18.4789 \\
\hline Spatial Median Filter & 13.1356 \\
\hline MSMF & 11.6743 \\
\hline Fused Image & 10.6356 \\
\hline
\end{tabular}


Edges define the boundaries between regions in an image, which helps with segmentation and object recognition. In order to better appraise the noise cancellation behavior of our filter from the point of view of human perception, an edge detection is performed for the fused image. Canny filter [3] is used for detection of edges in our "parrot" image. Figure 4 shows that our method significantly reduces impulse noise and the image details have been satisfactorily preserved.

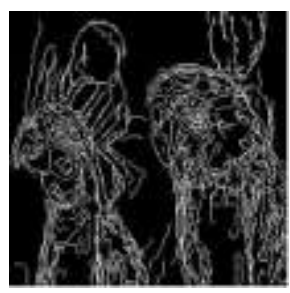

(a)

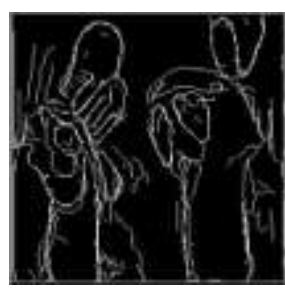

(b)

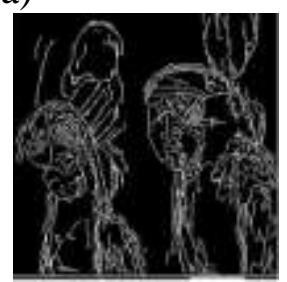

(c)

Figure 5. Edge detection using canny filter

a) Original noise free image

b) b) Noise image

c) c) Resultant image by our method

\section{CONCLUSION}

Image fusion technique for removing impulse noise in digital images is proposed. The image captured by the sensor undergoes filtering by different smoothing filters and the resultant images are fused to attain high quality image. This restoration of image data is very likely to find potential applications in a number of different areas such as electromagnetic imaging of objects, medical diagnostics, remote sensing, robotics, etc.

\section{REFERENCES}

[1] R. H. Laskar, B. Bhowmick, R. Biswas and S. Kar ," Removal of impulse noise from color image" , 2009 IEEE.

[2] James C. Church, Yixin Chen, and Stephen V. Rice, "A Spatial Median Filter for Noise Removal in Digital Images", 2008 IEEE.

[3] Ehsan Nadernejad, "Edge Detection Techniques: Evaluations and Comparisions" Applied Mathematical Sciences, Vol. 2, 2008, no. 31, $1507-1520$.

[4] S.Indu , Chaveli Ramesh, " A noise fading technique for images highly corrupted with impulse noise", Proceedings of the ICCTA07, IEEE.

[5] Damianos G. Karakos and Panos E. Trahanias, "Generalized Multichannel Image-Filtering Structures ",IEEE Transactions on image processing" , Vol. 6, No. 7, July 1997. 